\author{
O.L. Kapitanchuk, V.I. Teslenko
}

\title{
Modeling the Bimodal Behavior of Self-Repairing Optical Window Systems Prone to Brittle Failure
}

\author{
Bogolyubov Institute for Theoretical Physics, National Academy of Sciences of Ukraine, Kyiv 03680, Ukraine, \\ e-mail: alkapt@bitp.kiev.ua; vtes@bitp.kiev.ua
}

\begin{abstract}
Using an exact solution for transient state population of a three-stage absorbing Markov chain the problem of modeling the bimodal behavior of three window materials represented as some self-repairing optical systems prone to brittle failure is considered quantitatively. It is shown that simulated maximum failure probability density distributions can well describe the experimental data of biaxial tests on OFG, CVD-ZnSe and a-plane sapphire ceramics. The conclusion is made that the competitive advantage of these materials grows in proportion to their distribution widths.
\end{abstract}

Keywords: optical window systems, brittleness, failure susceptibility, competitive advantage.

Article acted received 15.07.2019; accepted for publication 15.09.2019.

\section{Introduction}

Natural and artificial systems, from living organisms to real world natural systems to solid state optoelectronic materials to engineered composites, are susceptible to damage. Conventionally, the various types of damage processes are divided into two groups. The first group comprises the case of steady failures, which are extrinsically induced in the system and can so be detected and then repaired. In this case, the dependence of the stationary failure probability density function on the external stress applied to the system can be well described by the Weibull distribution [1]. Rather, the second group of damages does not relate to steady-state failures. Instead, it comprises the intrinsic nonstationary failures, which can spontaneously appear and disappear in the system with no external cause. In this case, describing the temporal behavior of system failures requires nonstationary approaches, for instance, based on the balance transport equations [2] and discrete absorbing Markov chains [3,4] or the stochastic Markov decision processes [5]. The advantage of nonstationary approaches is that they make possible providing a correct description of a many failure state of the system by specifying it in terms of occupation numbers associated with small cells in the representation of a single failure state space with further considering the dynamics of populations of only these single failure states in those cells. However, due to the great generality of such approaches, there is a lack of simplified analytical expressions for stress dependence of failure probability density functions in the transient-state case, similarly to those of the familiar Weibull two- and three-parameter probability density functions in the steady-state case, as well as a five-parameter combination of two independent Weibull distributions. Meanwhile, recently, we succeeded in explicitly solving the three-stage absorbing Markov chain problem [6]. (Solution of the more general four-stage seven-parametric problem is unknown). Hence, in this paper we use the five-parameter expression for transient state population, obtained in [6], to describe experimental dependences on tensile stress at brittle fracture of the failure probability density functions for three optical window materials, namely OFG, CVD$\mathrm{ZnSe}$ and sapphire that are transparent in the IR spectrum. Furthermore, we get the possibility to consider such materials as a kind of systems equipped with the self-repairing properties, which have now been a reality and gained successful applications in many domains from aerospace to civil engineering (e.g. [7, 8] and references therein). The defects in those systems are considered as constantly appearing. However, the appeared defects are regarded to be small enough so that all they do not grow into catastrophic large scale defects resulting in system damage, but disappear almost autonomously returning the system into its normal functioning state.

In the next section of the paper we present the model of a self-repairing system having the single failure-prone transient state and three failure-tolerant initial, transient 
and absorbing states. In the one-defect approximation, this model corresponds to the three-stage absorbing Markov chain, for which a solution for the timedependent state populations has been obtained in [6]. Hence, using a simple exponential strain-stress model of brittle fracture we are allowed to represent the failure probability density function in the form of explicit dependence on the tensile stress. In section II, this dependence is compared with the experimental data of biaxial tests on OFG, CVD-ZnSe and sapphire ceramics. Finally, in section III the results obtained are discussed and concluded.

\section{Theoretical model}

In the problem of quantitative evaluation of material systems prone to failures, most approaches to probabilistic failure prediction are usually based upon the assumption that the dominant defect (or a tractably small set of identical non-interacting defects of significant type) can be identified before the analysis. It is also assumed that, despite the different types of defects may occur in all possible occupation numbers, there is the mean field approximation, which replaces the actual surrounding of a defect by a locally averaged number of neighboring defects, such that a single significant defect will influence one and only one set of measurements. Consequently, a many defect state of the material system can be specified in terms of occupation numbers (populations of states) associated with small elementary cells in a single-defect state space. However, the use of conventional statistical mechanics approaches to the simplified analysis of the dynamics of such systems can directly be straightforward only in the equilibrium steady-state case or quasi-equilibrium near to steadystate case. Therefore, a more general case of far from equilibrium transient systems requires nonstationary analyses. One example of such analyses is based on the concept of linear multistage absorbing Markov chain [4, 5]. In this concept, different states of a chain in the single-defect approximation can be associated with the states of the material system, which are occupied by the defect. Thus, defining for an absorbing Markov chain the number of its states and the probabilities of transitions between states (transition rate constants), and given initial conditions, we can solve the Markov chain problem for the nonstationary nonequilibrium state populations, which provides the possibility to use these in evaluating the behavior of system failures. However, to do this it is constructive to have the explicit expressions for nonstationary populations at hand. Due to [6] that is only possible for a linear absorbing Markov chain with not more than the three kinetic stages, which case can be depicted as follows

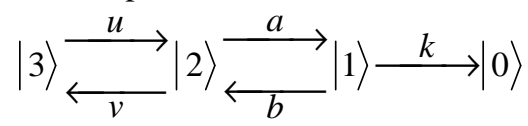

Here $\mid 3>$ is the initial failure-tolerant state, $\mid 2>$ is the transient failure-prone state, $\mid 1>$ is the intermediate failure-tolerant state, $\mid 0>$ is the absorbing failuretolerant state, and $u, v, a, b, k$ are the rate constants for between-state transitions directed with respective arrows. Note that all rate constant parameters are linearly independent and therefore form a complete fiveparameter set of the problem (1). This means that we can freely adjust their possible values by taking them from the intervals $[0, \infty)$ of numbers in some arbitrary dimensionless rate units.

The time evolution of Markov chain (1) is described by the following system of kinetic equations

$$
\left\{\begin{array}{l}
\&(t)=k p_{1}(t) ; \\
(t)=-(b+k) p_{1}(t)+a p_{2}(t) ; \\
(t)=b p_{1}(t)-(v+a) p_{2}(t)+u p_{3}(t) ; \\
\alpha(t)=v p_{2}(t)-u p_{3}(t) ;
\end{array}\right.
$$

written with respect to the populations $p_{i}(t)$ of different states $\mid i=0,1,2,3>$, with letting state $\mid 2>$ to be responsible for system failure. For the simplest initial condition

$$
p_{3}(0)=1 ; p_{2}(0)=p_{1}(0)=p_{0}(0)=0
$$

typical for nondestructive biaxial testing of window materials [9], the solution of (2) for $p_{2}(t)$ due to [6] reads

$$
p_{2}(t)=u \sum_{i} \frac{b+k-\lambda_{i}}{\prod_{j \neq i}\left(\lambda_{i}-\lambda_{j}\right)} \exp \left(-\lambda_{i} t\right)
$$

Here the exponents $\lambda_{n, m=1,2,3}$ under summation and product signs are the system eigenvalues that correspond to the non-negative Debye relaxation rates obeying the characteristic equation (see [6]):

$$
\lambda^{3}-\lambda^{2}(u+v+a+b+k)+\lambda[(u+v)(b+k)+a(u+k)]-u a k=0
$$

So, the time-dependent population $p_{2}(t)$ (4) of failure-prone state $\mid 2>$ of a Markov chain (1) represents an exponential temporal rise and decay pattern composed of three relaxation modes. Every mode, given the initial conditions (3), adds the particular contribution, differing in its eigenvalue, amplitude and sign. Since generally the time dependence of (4) shows an increase, peak and decline without oscillations, we are allowed to find the maximum of the population

$$
\bar{p}_{2}=p_{2}\left(t_{2}^{p e a k}\right)
$$

at the peak time moment $t=t_{2}^{\text {peak }}$ being a nontrivial solution of transcendent equation

$$
\delta(t)=0
$$

and then associate that maximum of population with the 
distribution of failure in the worst case.

In order to model the possible effect of the stress on the maximum failure probability distribution (6) we should take into account that, in the one-defect approximation, the actual state $|3\rangle$, in which the defect is initially initiated without causing the system failure, may contain the great number $N$ of different substates $\left|3_{n=1, \ldots, N}\right\rangle$. Since the transition rate constants in the various types of macromolecules inversely depend on the numbers of substates accessible for forward reactions [10], we expect that the external stress which can change such numbers will change the rate $u$ in (1) in the same fashion. If the tensile stress $\sigma$, normally used in a single measurement testing [9], is the factor which compresses the substate space of state $|3\rangle$, such that to decrease the number $N$ of its failure-tolerant substates from $N_{0} \sim \exp \left(-S_{0}\right) \quad$ to $\quad N_{\sigma} \sim \exp \left(-S_{\sigma}\right)<N_{0}, \quad$ or, equivalently, increase its effective (negative) thermodynamic entropy from $S_{0}$ to $S_{\sigma}>S_{0}$, then a dimensionless output rate $u / u_{\infty}$, where $u_{\infty}$ is the limiting rate for a closed-packed arrangement of substates $\mid 3_{n=1, \ldots, N_{\sigma}}>$ attained in the limit of infinite dimensionless stress $\sigma / \sigma_{0} \rightarrow \infty$, will be defined by the exponential relation

$$
u / u_{\infty}=\exp \left(\sigma / \sigma_{0}\right)-1
$$

The reason for this relation is that there is a typical situation for many optical window systems to contain defects in the form of cracks. In this case, the sharp edge of a crack will be the region of very high stress. Therefore, the dependence of reduced stress $\sigma / \sigma_{0}$ on reduced strain $1+x / x_{\infty}$ is not linear, such as away from a crack with the bulk parameters $\sigma$ and $x$ related by a Young's modulus E according to Hooke's law as $\sigma=\mathrm{E} x$ in the one-dimensional case, but logarithmic

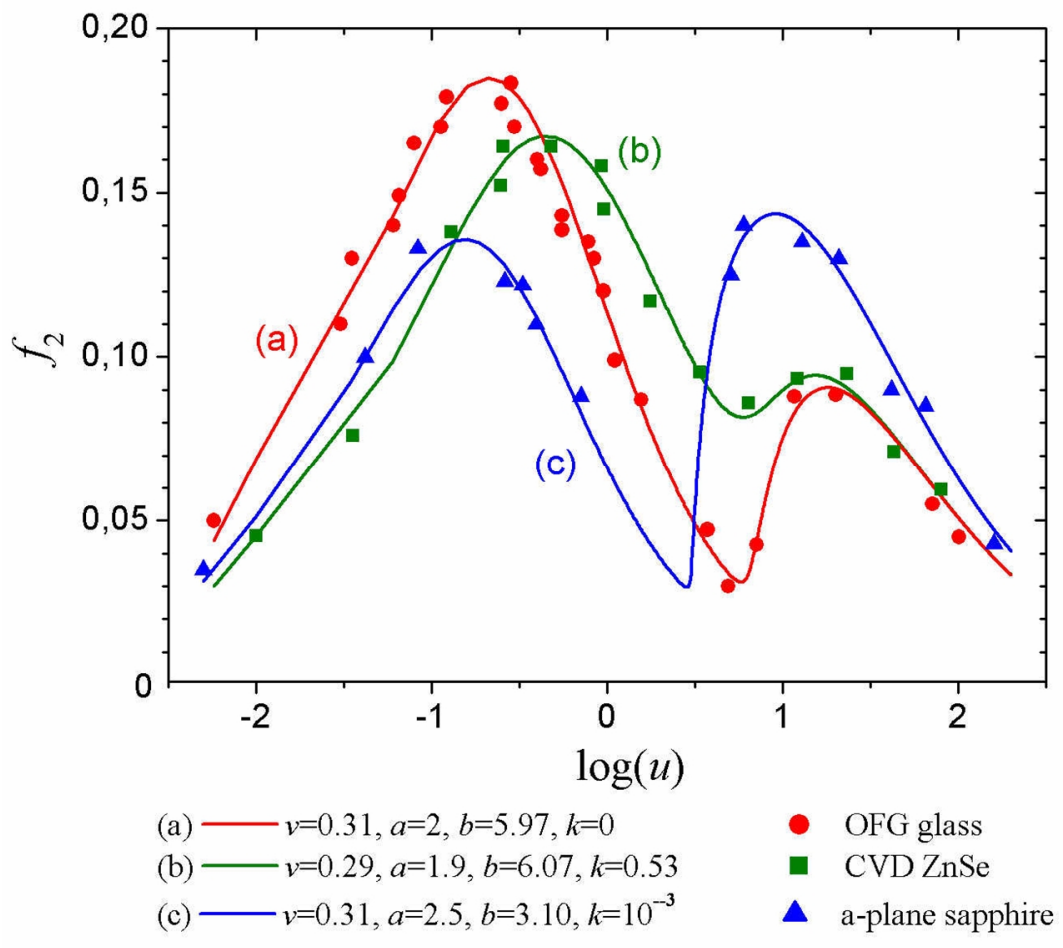

Fig. 1. The density distributions $f_{2}(\log u)$ (curves) simulated from (11) with the different rate constant parameters $v, a, b, k$ (given in inset in inverse time units). Experimental data (symbols) are adapted from Ref. 9 for three optical window materials. Figures: (a) oxyfluoride glass (circles), (b) CVD ZnSe (squares), and (c) aplane sapphire (triangles) correspond to the cases 1, 2, and 3 in Tab. 1, respectively.

Table 1

Values of parameters for density distributions in Fig. 1

\begin{tabular}{|c|c|c|c|c|c|c|c|}
\hline \multirow{2}{*}{ Case № } & \multirow{2}{*}{$\begin{array}{c}\text { Case-type } \\
\text { Classification }\end{array}$} & $v$ & $a$ & $b$ & $k$ & reduced mean peak & full width \\
\cline { 3 - 8 } & OFG & 0.31 & 2.0 & 5.97 & 0 & 0.153 & 3.534 \\
\hline 1. & CVD ZnSe & 0.29 & 1.9 & 6.07 & 0.53 & 0.149 & 3.219 \\
\hline 2. & a-plane sapphire & 0.31 & 2.5 & 3.10 & $10^{-3}$ & 0.139 & 3.685 \\
\hline 3. & & & & & & \\
\hline
\end{tabular}


$\sigma / \sigma_{0}=\log \left(1+x / x_{\infty}\right)$ yielding $\sigma \approx\left(\sigma_{0} / x_{\infty}\right) x$ only at $x<<x_{\infty}$. This is a simplest model for the materials, in which the hereditary properties and hysteresis effects are not taken into account [11]. Moreover, in a single failureresponse testing typical of window materials [9], the role of the reduced strain is played by the forward rate constant of transition in a Markov chain (1), that is, between its initial failure-tolerant state $|3\rangle$ and the nearest failure-prone state $|2\rangle$, to which the defect is transiently displaced during the action of applied stress. In general, such a transition rate constant depends exponentially on the free energy difference between these states, which includes an external stress as log of the number of substates of the initial failure-tolerant state |3> $[6,12,13]$. Therefore, the explanation of relation (8) is quite substantiated. Furthermore, since usually $\sigma>\sigma_{0}$, we can use in the sequel the simpler version of relation (8) in reduced logarithmic form

$$
\sigma=\log u
$$

as a single means for modeling the possible stress dependence of the corresponding desired functions

$$
\bar{p}_{2}=\bar{p}_{2}(\ln u)
$$

and

$$
\frac{\partial}{\partial \ln u} \bar{p}_{2}(\ln u)=f_{2}(\ln u)
$$

defining the failure cumulative distribution and probability density distribution, respectively.

\section{Comparison with experiment}

In the study of brittle fracture processes in ceramics and related composites, comparing the data of experiments on biaxial tensile testing for materials tested in the non-destructive measurements or single event damages with the theoretically simulated cumulative distributions and probability density functions constitutes a strong concern about the applicability of the predicted failure behavior [9, 14-17]. In this section we compare the failure probability density distribution (11) with the experimental data adapted from [9] for three IRtransmitting window materials, that is, OFG, CVD-ZnSe and sapphire, operating in the different margins of safety (see also [17-20]). The results of comparison are depicted in Fig. 1. As seen, there is a sufficiently good agreement between the theory and the experiment, particularly when concerning the bimodal behaviors of experimental bipeaked patterns of the density distributions. The respective sigmoidal patterns of the cumulative distributions have been considered elsewhere [21].

Hence, when characterizing the maximal damageability of nonstationary failure-prone state in a three-stage absorbing Markov chain (1) that models the transient temporal behavior of damage in a self-repairing damaged system, it is the case of avoiding the use of the bimodal five-parameter Weibull probability distribution has to be ad hoc introduced to better describe the data [20]. As is known, the Weibull distribution belongs to the family of special equilibrium distributions whose derivation, due to the Fisher-Tippett-Gnedenko theorem, is based on asymptotic arguments in the problem of distributing extremum values of the large-sample sequences of independent random variables with the same probability distribution as the sample size increases. On the contrary, the five-parameter Markov chain probability distribution (11) is essentially nonequilibrium one and has the bimodal property in itself. Furthermore, just the framework of a three-stage absorbing Markov chain provides a clear physical sense for failed state and equips the temporal behavior of a self-repairing damaged system with a transient failure model in a single defect approximation. This does not mean that if such an approximation is not valid, it will not be possible to describe experimental data in a simple fashion, but only that using the model of nonequilibrium absorbing Markov chain may be more advantageous than the use of the equilibrium Weibull distribution. This is even more obvious, since the mathematical model of stochastic Markov chain processes is straightforward in the formalism of balance-like (master) equations for chain state populations [22] that in turn is a direct consequence of mapping the evolution of a nonequilibrium quantum system weakly coupled with the equilibrium environment onto the population state space in the one-particle approximation (for more details see e.g. $[23,24])$.

\section{III.Discussion and conclusions}

In this work, based on analytical solution (4) for the temporal behavior of population $p_{2}(t)$ of failure-prone state $\mid 2>$ of a three-stage absorbing Markov chain (1), with three other its states $|3>| 1>$, and $\mid 0>$ being failure-tolerant, we describe the failure probability density function $f_{2}(\sigma)$ (11) in its dependence of the applied stress $\sigma=\log u$ (9), logarithmically proportional to the failure rate $u$ input to the state $\mid 2>$. Our main concern is to compare this dependence with the experimental density distributions of tensile testing data for some optical window materials. Choosing these as OFG, CVD-ZnSe and sapphire, we find a correspondence between theoretical predictions and experimental findings (see Fig. 1). Additionally, we account for the fact that the found density distributions, though all being bimodal, are peaked to different amplitudes for the different modes and have different full widths on the reduced mean half maximum. This allows us to make a conclusion that, given the tolerances with respect to the cumulative response of the population of failure-prone state on limiting stresses, the material system with the larger margin of safety in $\log$ of the stress space, i.e., with the wider density distribution which corresponds to the smaller slope of the stressresponse curve, exhibits lower sensibility to failure and therefore higher competitive advantage toward other materials considered as competitors [25]. The values of respective amplitudes and widths for the density distributions, simulated for three different optical window materials by Eqs. (4)-(11) and presented in Fig.1, as well as the corresponding values of kinetic 
parameters used for simulation, are summarized in Tab.1. These substantiate the above conclusion, as well as coincide with a similar conclusion of Ref. 21, though, made on the different reasons of using the concept of competitiveness coefficient. The latter is known to be the analog to the indicator of the normal stability of the system and its capability to perform reliably and safely on the standardized testing or potentially deleterious impacts without failure, being complementary to its cooperativity [21].

Thus, basing on the numerical results obtained by simulations we can conclude that among three IRtransmitting window materials analyzed in this work, the a-plane sapphire exhibits advanced performance revealing a higher competitive advantage regarding other two materials, while the CVD ZnSe performs poorly compared to the OFG glass. This conclusion corresponds well to the experimental observations provided in [9].

\section{Conflicts of interest}

Authors declare that there are no conflicts of interest between them.

\section{Acknowledgments}

The present work was partially supported by The National Academy of Sciences of Ukraine (project No. 0116U002067).

Kapitanchuk O.L. - PhD, Senior Research Fellow; Teslenko V.I. - Doctor of Science (Engineering), Leading Research Fellow.

[1] D.N.P. Murthy, M. Xie and R. Jiang, Weibull Models (John Wiley\& Sons, New York, 2004).

[2] B. Novakovic and I. Knezevic, Quantum master equations in electronic transport, in: Nano-Electronic Devices: Semiclassical and Quantum Transport Modeling, edited by D. Vasileska and S. M. Goodnic (Springer, New York, 2011). P. 249.

[3] K.S. Trivedi, Probability and Statistics with Reliability, Queuing, and Computer Science Applications (John Wiley \& Sons, Hoboken, NJ, 2016).

[4] D.M. Nicol, W.H. Sanders and K.S. Trivedi, IEEE Trans. Depend. Secure Comput. 1, 48 (2004) (doi: 10.1109/TDSC.2004.11).

[5] M.I. Puterman, Markov Decision Processes: Discrete Stochastic Dynamic Programming (Wiley, Hoboken, NJ, USA, 2014).

[6] O.L. Kapitanchuk, O.M. Marchenko and V.I. Teslenko, Chem. Phys. 472, 249 (2016) (doi: 10.1016/j.chemphys.2016.03.007).

[7] H. Fisher, Nat. Sci. 2, 873-901 (2010) (doi: 10.4236/ns.2010.28110).

[8] U. Lafont, H. van Zeijl and S. van der Zwaag, Microelectronics Reliab. 52, 71 (2012).

[9] C.A. Klein, Proc. SPIE 7504, 75040K (2009) (doi: 10.1117/12.836920).

[10] H. Frauenfelder and P.G. Wolynes, Science 229, 337 (1985) (doi: 10.1126/science.4012322).

[11] O.V. Rudenko, Usp. Fiz. Nauk 176, 77 (2006). [Phys. Usp. 49, 69 (2006).]

[12] V.I. Teslenko, O.L. Kapitanchuk and Y. Zhao, Chin. Phys. B 24, 028702 (2015) (doi: 10.1088/16741056/24/2/028702).

[13] E. Petrov and V. Teslenko, Nanobiophysics: Fundamentals and Applications, edited by V. A. Karachevtsev, chapter 9, p.267 (Pan Stanford Publishing, Singapore, 2016) (doi: 10.1201/b20480).

[14] J.P. Berry, Nature 212, 20 (1966) (doi: 10.1038/212020a0).

[15] B. Tsoi, E. M. Kartashov and V. V. Shevelev, The Statistical Nature of Strength and Lifetime in Polymer Films and Fibers (VSP, Zeist, The Netherlands, 2004). [B. Tsoi, É. M. Kartashov and V. V. Shevelev, Strength and Fracture of Polymer Films and Fibers (Khimiya, Moscow, 1999)].

[16] C.A. Klein, Opt. Eng. 50(2), 023402 (2011) (doi: 10.1117/1.3541804).

[17] C.A. Klein and R. P. Miller, Proc. SPIE 4375, 2411 (2001) (doi: 10.1117/12.439181).

[18] CA. Klein, J. Appl. Phys. 96, 3172 (2004) (doi: 10.1063/1.1782272).

[19] C. A. Klein, Proc. SPIE 8016, 80160J (2011) (doi: 10.1117/12.883019).

[20] V.I. Teslenko and O.L. Kapitanchuk, Mod. Phys. Lett. B 32, 1850022 (2018) (doi: 10.1142/S0217984918500227).

[21] A.N. Gorban, Entropy 16, 2408-2432 (2014) (doi:10.3390/e16052408).

[22] V. I. Teslenko and O. L. Kapitanchuk, J. Phys. Stud. 19, 1001 (2015).

[23] V.I. Teslenko and O. L. Kapitanchuk, Acta Phys. Polon. 49, 1581 (2018) (doi: 10.5506/APhysPolB.49.1581).

[24] C.A. Klein, Opt. Eng. 37, 2826 (1998) (doi: 10.1117/1.601820). 


\title{
О.Л. Капітанчук, В.І. Тесленко
}

\section{Моделювання бімодальної поведінки самовідновлювальних оптично прозорих систем, схильних до крихкого руйнування}

\author{
Інститут теоретичної фізики ім. М.М. Боголюбова, Національна Академія Наук України, Київ 03680, \\ Україна,e-mail: alkapt@bitp.kiev.ua; vtes@bitp.kiev.ua
}

\begin{abstract}
Проблема моделювання бімодальної поведінки прозорих матеріалів, що можуть бути представленими у вигляді деяких самовідновлювальних оптичних систем, схильних до крихкого руйнування, розглядається кількісно з використанням точного розв'язку для заселеності перехідного стану тристадійного абсорбуючого ланцюга Маркова. Показано, що зсимульовані густини розподілів максимальних ймовірностей руйнування можуть добре описувати експериментальні данні з біаксіального розтягнення керамік OFG, CVD-ZnSe i сапфіру в а-площині. Зроблено висновок, що конкурентна перевага цих матеріалів росте пропорційно ширині їх розподілів.
\end{abstract}

Ключові слова: оптично прозорі системи, крихкість, схильність до руйнування, конкурентна перевага. 